\title{
The Enskog theory for classical vibrational energy relaxation in fluids with continuous potentials
}

\author{
Biman Bagchia) and Goundla Srinivas \\ Solid State and Structural Chemistry Unit, Indian Institute of Science, Bangalore 560 012, India \\ Kunimasa Miyazaki ${ }^{\text {b) }}$ \\ IRI, Delft University of Technology, 2629 JB Delft, The Netherlands
}

(Received 22 March 2001; accepted 20 June 2001)

The recently developed Enskog theory for binary friction for fluids with continuous potentials, such as the Lennard-Jones, has been extended to calculate the frequency dependence of this friction, $\zeta_{E}(\omega)$. This $\zeta_{E}(\omega)$ is then applied to study vibrational energy relaxation of low-frequency modes via the Landau-Teller expression. The agreement with simulation results is found to be satisfactory. In the present approach we provide an exact prescription for the binary friction and thus remove a lacuna in this area. $\zeta_{E}(\omega)$ shows an interesting structure with a hump at low frequency, the signature of which has already been seen in many simulation studies. (C) 2001 American Institute of Physics.

[DOI: $10.1063 / 1.1392360]$

\section{INTRODUCTION}

The study of vibrational energy relaxation (VER) forms an essential part of physical chemistry and has a long history. ${ }^{1,2}$ This long interest in VER comes from the critical role that it plays in chemical reactions. However, it was not easy to obtain information about the rates of vibrational energy relaxation in a bond. The study of VER gained momentum after laser spectroscopy allowed a measurement of the population of vibrational levels. Theoretical studies on vibrational energy relaxation have mostly been carried out by invoking two basic models - the isolated binary collision (IBC) model $^{3}$ in which the collision frequency is modified by the liquid structure, and the weak coupling model, ${ }^{1}$ where the vibrational motion of the molecule weakly couples to the rest (translational and rotational) degrees of freedom so that a perturbative technique can be employed.

The IBC model was developed by Herzfeld et al. ${ }^{3}$ In this model, one starts with the assumption that the VER rate for a two level system can be given by

$$
\tau_{i j}^{-1}=P_{i j} \tau_{c}^{-1},
$$

where $\tau_{c}^{-1}$ is the collision frequency and $P_{i j}$ is the probability per collision that a transition from level " $i$ " to level " $j$ " will take place. $P_{i j}$ is independent of density but does depend on temperature whereas $\tau_{c}$ depends on both these state parameters. In the gas phase, $\tau_{c}$ can be obtained from kinetic theory, but it is difficult to obtain in the condensed phase. If the colliding molecules are approximated by effective hard spheres with radius $\sigma$, then an expression for the collision frequency can be obtained from the Enskog theory, which gives the frequency as proportional to $g(\sigma)$, where $g(\sigma)$ is the value of the radial distribution function at contact. $\tau_{c}$ is also proportional to the friction and hence to the viscosity of the gas. Thus, the IBC model with Enskog collision fre-

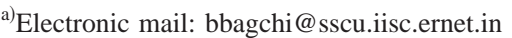

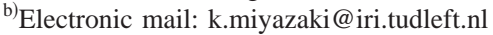

quency predicts a rate proportional to the viscosity of the gas. The point of interest here is that there does not exist any reliable calculation of the binary friction in a dense liquid within the Enskog theory for a continuous potential.

The second line of approach considered the vibrational energy relaxation as a classical process where energy is dissipated to the medium by the usual frictional process. Then, one can adopt a stochastic approach. For VER involving low frequencies, it is reasonable to assume that the vibration is harmonic. Under this condition, one can write a generalized Langevin equation of motion for the normal coordinate $Q(t)$ as $^{4-6}$

$$
\begin{aligned}
\mu \ddot{Q}(t)= & -\mu \bar{\omega}^{2} Q(t)-\int_{-\infty}^{t} \mathrm{~d} t^{\prime} \zeta_{\text {bond }}\left(t-t^{\prime}\right) \dot{Q}\left(t^{\prime}\right) \\
& +R_{Q}(t)
\end{aligned}
$$

where $\mu$ is the reduced mass of the diatomic comprising the vibrating bond and $\bar{\omega}$ is the renormalized bond frequency that is shifted from the bare bond frequency due to the presence of solvent molecules, ${ }^{6} \zeta_{\text {bond }}(t)$ is the time-dependent bond friction coefficient, and $R_{Q}(t)$ is the random force related to $\zeta_{\text {bond }}(t)$ by the fluctuation-dissipation theorem. The following important observation is made at this point. Even at as low a bond frequency as $100 \mathrm{~cm}^{-1}$, the solvent frictional force on the bond is very small. Thus, while the zero frequency friction can be large, the relaxation of $Q$ probed at large frequency can be in the underdamped limit.

The rate of the VER, $1 / T_{1}$, of a classical oscillator is given by the simple Landau-Teller expression, ${ }^{4-7}$

$$
\frac{1}{T_{1}}=\frac{\zeta_{\text {bond }}(\bar{\omega})}{\mu},
$$

where $\zeta_{\text {bond }}(\omega)$ is the Fourier-Laplace transform of $\zeta_{\text {bond }}(t)$. This friction is responsible for population redistribution in vibrational levels since energy dissipates through this friction. 
If the cross-correlation between the forces on two atoms is neglected, one can approximate the bond friction coefficient $\zeta_{\text {bond }}(\bar{\omega})$ by that of the single atom (free-draining approximation). Especially, for the homonuclear diatomic consisting of the same atoms, it is given by

$$
\zeta_{\text {bond }}(\bar{\omega})=\frac{\zeta(\bar{\omega})}{2}
$$

where $\zeta(\bar{\omega})$ is the friction experienced by one of the atoms of the vibrating homonuclear diatomic. Equation (4) was also used by Oxtoby in his theory of vibrational dephasing. ${ }^{2}$ Equation (4) gives a simple way to relate the solvent dynamic response to the VER rate. The validity of the freedraining approximation is systematically examined by Berne et al. using molecular dynamics simulations ${ }^{5}$ and the Zwanzig-Bixon model based on the continuous hydrodynamic equation, ${ }^{8}$ and it is found that the approximation is not verified for a short bond length. However, this approximation is still semiquantitatively reliable, and so is the LandauTeller expression, at least for the low-frequency mode. Therefore, the success of the weak coupling method depends on an accurate frequency-dependent friction. However, an analytic calculation of frequency-dependent friction has been a difficult task and several different approximations have been used. The most successful is the scheme used by Egorov and Skinner, ${ }^{4}$ who fitted the short time expansion to a $\cos (a t) / \cosh (b t)$ form, where the constants $a$ and $b$ are obtained from the second and fourth time derivatives of the force-force time correlation function at time $t=0$. The other method is the instantaneous normal mode (INM) analysis, which has also been fairly successful in getting the highfrequency response of the liquid. ${ }^{6}$

In a recent paper ${ }^{9}$ (hereafter refer to as paper I), we have presented a novel method to obtain an Enskog level description of binary friction of continuous potential. Numerical implementation of this new method gave excellent agreement of the time-dependent friction and self-diffusion coefficient with simulation results.

In this paper, this new Enskog theory is used to calculate the frequency-dependent binary friction for fluids interacting with the Lennard-Jones potential. We use this friction to calculate the rate of the vibrational energy relaxation and compared the theoretical result with available simulation data. The agreement between the theory and simulation is found to be satisfactory. In a sense, this work can be regarded as an amalgamation of IBC and the Landau-Teller approach to VER.

Another interesting aspect of this work is the fact that the frequency-dependent binary friction $\zeta_{E}(\omega)$ shows an interesting frequency dependence with a hump at low frequency, which has already been seen in many simulation studies. We regard this hump describing the crossover frequency below which the collective effects become important.

This paper is organized as follows. In the next section, the formulation of the binary frequency-dependent friction under an Enskog approximation is summarized. In Sec. III, we present results and Sec. IV contains concluding remarks.

\section{FORMAL THEORY}

The frequency-dependent friction from the Enskog theory is rather involved. The details of the theory are available in paper I. In this section, we present the essential parts.

We shall consider an atom in a simple fluid that interacts with other identical atoms with a pairwise potential $\phi(r)$. The motion of an atom in a gas or a liquid is often described in terms of the velocity autocorrelation function, $\psi(t)$ $=\langle\mathbf{v}(t) \cdot \mathbf{v}(0)\rangle$, where $\mathbf{v}(t)$ is the velocity of the atom at time $t$ and $\langle\cdots\rangle$ is the equilibrium ensemble average. Its time evolution is described by a generalized Langevin equation..$^{10}$ If one takes Laplace transform in time, this equation is written as

$$
[m z+\zeta(z)] \psi(z)=\psi(t=0),
$$

where $m$ is the mass of the atom, $\psi(z)$ denotes the Laplace transformation of $\psi(t)$, and $\zeta(z)$ is the frequency-dependent friction coefficient or the memory kernel. The friction coefficient is given by the Green-Kubo formula as ${ }^{10}$

$$
\zeta(z)=\frac{1}{k_{\mathrm{B}} T} \int_{0}^{\infty} \mathrm{d} t e^{-z t}\left\langle F_{x}(t) F_{x}(0)\right\rangle .
$$

Here $\mathbf{F}(t)$ is the force between the solute and surrounding solvents at a time $t, k_{\mathrm{B}}$ is the Boltzmann constant, and $T$ is the absolute temperature. Note that the time evolution of $\mathbf{F}(t)$ in Eq. (6) is not driven by the usual Liouville operator, but given by

$$
\mathbf{F}(t) \equiv \exp \left[i \mathcal{Q} L_{N} t\right] \mathbf{F}(0),
$$

where $i L_{N}$ is the Liouville operator for the $N$-particle system and $\mathcal{Q}=1-\mathcal{P}$ and $\mathcal{P}$ is a projection operator defined by $\mathcal{P} *$ $=\left\langle * v_{x}\right\rangle v_{x} /\left\langle v_{x}^{2}\right\rangle$. This equation can be established by using the Mori-Zwanzig projection-operator method. ${ }^{10,11}$ Equation (6) can be rewritten as

$$
\begin{aligned}
\zeta(z)= & \frac{1}{V k_{\mathrm{B}} T} \int \mathrm{d} 1 \int \mathrm{d} 2 \int \mathrm{d} 3 \int \mathrm{d} 4 \frac{\partial \phi\left(r_{12}\right)}{\partial x_{1}} \\
& \times G(1,2 ; 3,4 ; z) \frac{\partial \phi\left(r_{34}\right)}{\partial x_{3}},
\end{aligned}
$$

where $V$ is the volume of the system, $r_{12}=\left|\mathbf{r}_{1}-\mathbf{r}_{2}\right|$, and $G(1,2 ; 3,4 ; z)=V\left\langle\left[\left(z-i \mathcal{Q} L_{N}\right)^{-1} f_{2}(1,2)\right] f_{2}(3,4)\right\rangle \quad$ is the four-points propagator that describes the time evolution of a particle interacting with other particles. ${ }^{12}$ In this expression,

$$
f_{2}(1,2) \equiv \delta\left(1-q_{1}\right) \sum_{i=2}^{N} \delta\left(2-q_{i}\right)
$$

is the two-particle phase-space distribution function, where $1=\left(\mathbf{r}_{1}, \mathbf{p}_{1}\right)$ and $q_{i}=\left(\mathbf{R}_{i}, \mathbf{P}_{i}\right) \quad(i=1,2, \ldots, N)$ are the phasespace coordinates and the actual coordinates of the particles, respectively. This representation makes it easy to understand how the binary collision approximation comes to play a role. Mazenko ${ }^{13}$ showed that the equation for $G(1,2 ; 3,4 ; z)$ can be expressed without invoking the projected Liouville operator $i \mathcal{Q} L_{N}$. Keeping only the binary collision term in his expression, we have

$$
\left[z+i L_{2}(1,2)\right] G(1,2 ; 3,4 ; z)=G(1,2 ; 3,4 ; t=0),
$$


where $i L_{2}(1,2)$ is the Liouville operator for the two-body system. Therefore, the friction coefficient under the binary collision approximation, which we shall refer to as $\zeta_{E}(z)$, can be written as

$$
\begin{aligned}
\zeta_{E}(z)= & \frac{1}{V k_{\mathrm{B}} T} \int \mathrm{d} 1 \int \mathrm{d} 2 \int \mathrm{d} 3 \int \mathrm{d} 4 \frac{\partial \phi\left(r_{12}\right)}{\partial x_{1}} \\
& \times\left[z+i L_{2}(1,2)\right]^{-1} G(1,2 ; 3,4 ; t=0) \frac{\partial \phi\left(r_{34}\right)}{\partial x_{3}} .
\end{aligned}
$$

Furthermore, one may use the following identity:

$$
\begin{gathered}
\int \mathrm{d} 3 \int \mathrm{d} 4 G(1,2 ; 3,4 ; t=0) \frac{\partial \phi\left(r_{34}\right)}{\partial x_{3}} \\
=-\rho k_{\mathrm{B}} T f_{0}\left(p_{1}\right) f_{0}\left(p_{2}\right) \frac{\partial g\left(r_{12}\right)}{\partial x_{1}},
\end{gathered}
$$

where $f_{0}(p)=\left(2 \pi m k_{\mathrm{B}} T\right)^{-3 / 2} \exp \left(-p^{2} / 2 m k_{\mathrm{B}} T\right)$ is the Maxwell distribution function, $\rho$ is the number density of the system, and $g(r)$ is the radial distribution function. Then, we may simplify the expression as

$$
\begin{aligned}
\zeta_{E}(z)= & -\frac{\rho}{V} \int \mathrm{d} 1 \int \mathrm{d} 2 f_{0}\left(p_{1}\right) f_{0}\left(p_{2}\right) \frac{\partial g\left(r_{12}\right)}{\partial x_{1}} \\
& \times\left[z-i L_{2}(1,2)\right]^{-1} \frac{\partial \phi\left(r_{12}\right)}{\partial x_{1}} .
\end{aligned}
$$

Equation (11) can be further simplified by introducing the center-of-mass coordinate and relative coordinates. Integrating over the irrelevant center-of-mass coordinate, we arrive at

$$
\begin{aligned}
\zeta_{E}(z)= & -\rho \int d \mathbf{p} \int d \mathbf{r} f_{0}^{\mathrm{red}}(p) \frac{\partial g(r)}{\partial x} \\
& \times[z-i L(\mathbf{r}, \mathbf{p})]^{-1} \frac{\partial \phi(r)}{\partial x},
\end{aligned}
$$

where

$$
i L(\mathbf{r}, \mathbf{p})=\frac{2 \mathbf{p}}{m} \cdot \frac{\partial}{\partial \mathbf{p}}-\frac{\partial \phi(r)}{\partial \mathbf{r}} \cdot \frac{\partial}{\partial \mathbf{p}}
$$

is the Liouville operator for the relative coordinate $(\mathbf{r}, \mathbf{p})$ and $f_{0}^{\mathrm{red}}(p)=\left(\pi m k_{\mathrm{B}} T\right)^{-3 / 2} \exp \left(-p^{2} / m k_{\mathrm{B}} T\right)$. In the real time representation, Eq. (12) becomes

$$
\zeta_{E}(t)=-\rho \int d \mathbf{p} \int d \mathbf{r} f_{0}^{\mathrm{red}}(p) \frac{\partial g(r)}{\partial x} \frac{\partial \phi\left(r_{t}\right)}{\partial x_{t}},
$$

where $\mathbf{r}_{t}=e^{i L(\mathbf{r}, \mathbf{p}) t} \mathbf{r}$ is the relative position at time $t$ for the two colliding particle systems for a given initial condition $\mathbf{r}_{t=0}=\mathbf{r}$. Equations (12) and (13) are the Enskog expressions for the frequency- and time-dependent friction coefficient, respectively, which are valid for arbitrary potentials.

It has been shown in paper I that (i) Eq. (13) satisfies the exact initial condition, i.e., $\zeta_{E}(t=0)=\zeta(t=0)$; (ii) Eq. (12) reduces to the well-known Chapman-Enskog expression for the friction ${ }^{14}$ in the low-density and low-frequency limit; and (iii) for the hard-sphere potential, Eq. (12) reduces to the well-known Enskog expression if one neglects contribution from the inhomogeneity of $g(r)$.
The time-dependent friction coefficient, Eq. (13), can be calculated, provided that the positions of all possible trajectories of the two particle system are known. This can be done easily by solving the equation of motion. We evaluate the positions $\left(r_{t}, \theta_{t}\right)$ at time $t$ in the polar coordinates for a given initial condition $(p, r, \theta)$, where we choose the direction of the velocity parallel to the polar axis. The problem can be reduced to the one-dimensional problem of the motion in the effective potential given by $\phi_{\text {eff }}(r)=\phi(r)+p^{2} b^{2} / m r^{2}$, where $b \equiv r \sin \theta$ is the impact parameter. The solution of the equation of motion is given by

$$
t=\int^{r_{t}} \mathrm{~d} r^{\prime} f\left(r^{\prime}\right), \quad \theta_{t}=\frac{2 b p}{m} \int^{r_{t}} \mathrm{~d} r^{\prime} \frac{f\left(r^{\prime}\right)}{r^{\prime 2}} .
$$

Here $f(r)=1 / \sqrt{4\left[E-\phi_{\text {eff }}(r)\right] / m}$, where $E$ is the total energy of the two particle system. $r_{t}$ for arbitrary $t$ is evaluated by solving the first equation of above equations. $\theta_{t}$ can be evaluated from the second equation using $r_{t}$ evaluated from the first. The constants of integration can be chosen such that they satisfy the initial condition.

\section{NUMERICAL RESULTS}

In this section, we shall consider a homonuclear diatomic molecule consist of identical atoms as the surrounding solvent atoms. The intermolecular potential is assumed to be given by the Lennard-Jones potential, defined by

$$
\phi(r)=4 \epsilon\left[\left(\frac{\sigma}{r}\right)^{12}-\left(\frac{\sigma}{r}\right)^{6}\right],
$$

where $\epsilon$ and $\sigma$ are the standard Lennard-Jones parameters.

First let us consider the frequency-dependent friction coefficient for a single solute atom. We calculate the timedependent friction [Eq. (13)], using $\mathbf{r}_{t}$ evaluated from Eq. (14) and, then transformed the result to the frequency representation using fast Fourier transform (FFT). In Fig. 1, we show the comparison between the calculated Enskog friction and the single-particle frequency-dependent friction obtained from the molecular dynamic simulation of Straub et al. ${ }^{15}$ for $\rho^{*} \equiv \rho \sigma^{3}=1.05$ and $T^{*} \equiv k_{\mathrm{B}} T / \epsilon=2.5$.

In the theoretical calculation, we have used $g(r)$ obtained both from the molecular dynamics simulations and from Percus-Yevick (PY) theory. In Fig. 1 we show theoretical results only with simulated $g(r)$ - the results with PY $-g(r)$ are not significantly different. The agreement between our theory and simulation is satisfactory at all frequencies above $50 \mathrm{~cm}^{-1}$.

Note that in view of the fact that Fig. 1 depicts the frequency dependence of pure binary friction, the hump on the low-frequency side acquires a special significance. This hump is also seen in simulation studies. One can regard this hump as the signature of a crossover. At frequencies below this hump, the collective effects like density or current relaxations become important.

The bond friction is obtained in terms of atomic friction by using Eq. (4). We present the calculated rate of the vibrational energy relaxation in Table I. The atomic mass and size of the diatomic solute are the same as those of an argon atom. In this table, the theoretical prediction is compared 


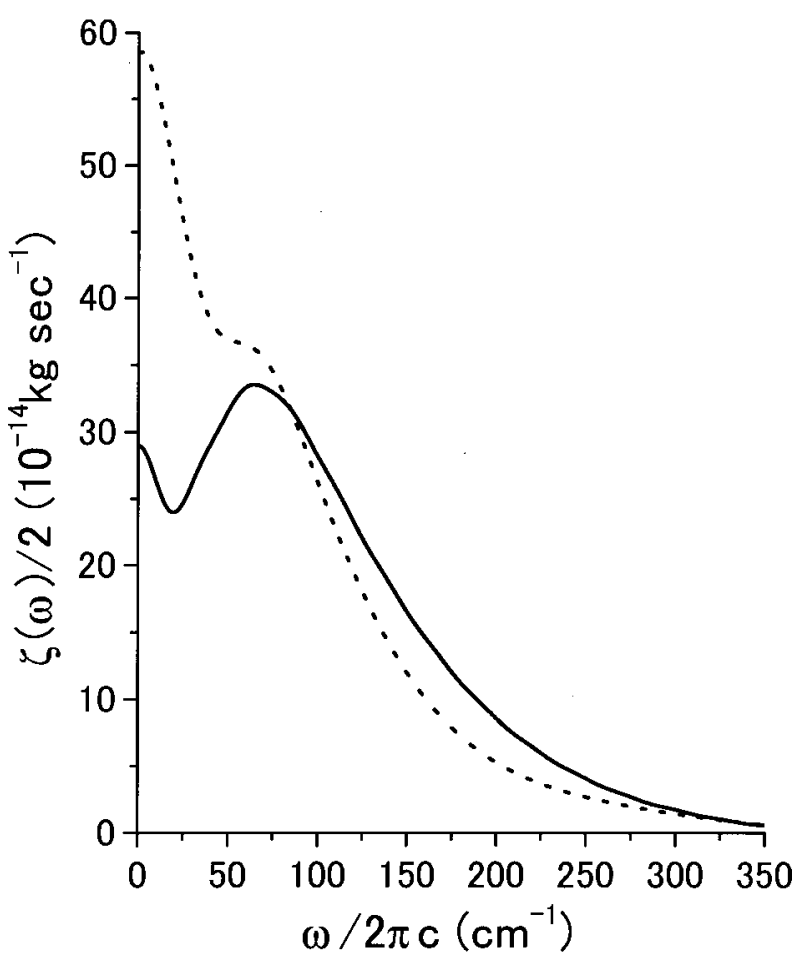

FIG. 1. Frequency-dependent friction for the solvent atom calculated from the Enskog theory [Eq. (13)] is compared with that obtained from MD simulation by Berne et al. (Ref. 15). The half-value $\zeta(\omega) / 2$ is plotted for $T^{*}=2.5$ and $\rho^{*}=1.05$. The parameters used are from argon atom; $\sigma_{\mathrm{Ar}}$ $=3.41 \AA, \epsilon / k_{\mathrm{B}}=120.0 \mathrm{~K}$, and $m_{\mathrm{Ar}}=39.5 \mathrm{amu}$. Solid line shows the result of Enskog theory by using the $g(r)$ obtained from the present MD simulation. The result of Straub et al. is shown by a dashed line.

both with the simulation results of Berne $e t a l .{ }^{5}$ and the INM predictions. ${ }^{6}$ In Ref. 5, $\zeta_{\text {bond }}(\bar{\omega})$ has been calculated. We have used Eq. (3) to evaluate $1 / T_{1}$. The agreement is semiquantitative. Note that Theory ${ }_{\mathrm{sim}}$ and Theory $\mathrm{PY}_{\mathrm{PY}}$ represent the results obtained by using the $g(r)$ obtained from the present MD simulation and PY scheme, respectively. It is heartening to see that a purely theoretical scheme [with PY- $g(r)]$ reproduces the results reasonably well.

Note that over the frequency range covered in Table I, the frequency varies approximately almost by a factor of 6 and the rate varies by more than an order of magnitude.

TABLE I. The vibrational energy relaxation rates calculated by using Eqs. (3) and (4) along with Eq. (13) of the text. Theory $y_{\text {sim }}$ and Theory $y_{P Y}$ represents the result obtained by using the $g(r)$ obtained from the present MD simulation and PY scheme, respectively. The system studied here is a homonuclear diatomic solute consist of the same atom as solvent atoms at $\rho^{*}$ $=1.05$ and $T^{*}=2.5$. The argon parameters given in Fig. 1 are used. For comparison, the simulation results of Berne et al. (Ref. 5) and the INM results of Stratt et al. (Ref. 6) are also shown in the same table.

\begin{tabular}{ccccc}
\hline \hline $\bar{\omega} / 2 \pi c\left(\mathrm{~cm}^{-1}\right)$ & $\begin{array}{c}1 / T_{1}\left(\mathrm{ps}^{-1}\right) \\
\left(\text { Theory }_{\text {sim }}\right)\end{array}$ & $\begin{array}{c}1 / T_{1}\left(\mathrm{ps}^{-1}\right) \\
\left(\text { Theory }_{\mathrm{PY}}\right)\end{array}$ & $\begin{array}{c}1 / T_{1}\left(\mathrm{ps}^{-1}\right) \\
(\text { simulations })\end{array}$ & $\begin{array}{c}1 / T_{1}\left(\mathrm{ps}^{-1}\right) \\
(\mathrm{INM})\end{array}$ \\
\hline 39.7 & 8.83 & 9.29 & 11.26 & 12.69 \\
68.7 & 10.19 & 10.77 & 9.64 & 12.5 \\
145.0 & 5.37 & 5.70 & 3.16 & 3.26 \\
221.6 & 1.93 & 1.70 & 0.99 & 0.05 \\
\hline \hline
\end{tabular}

Since the theory involves a series of approximations, like the use of a Landau-Teller approximation and the neglect of cross-correlations between the forces on the two atoms, the agreement can be considered satisfactory, and it can be surmised that the binary part alone is capable of explaining the rate of the vibrational energy relaxation. The agreement is satisfactory, even at low frequency, where frequencydependent friction clearly derives considerable contributions from collective dynamics.

\section{CONCLUDING REMARKS}

The comparison between the theoretical predictions and the simulation results shown in Table I seems to suggest that the binary part alone is capable of explaining the rate of the vibrational energy relaxation, at least semiquantitatively.

Note that, in the present work, we have assumed that there is no coupling between the translational and rotational motions. However, in real systems the coupling between the rotational and the translational motions may become rather important. The study of VER becomes even more challenging and interesting if a direct calculation of the rate of VER is carried out by using the Enskog method developed in paper I. In this future problem, one can address the role of rotational and vibrational coupling in an explicit way. Such a calculation may throw considerable light on VER in supercritical fluids.

\section{ACKNOWLEDGMENTS}

The authors thank Professor I. M. de Schepper for encouragement and an enlightening discussion. One of the authors (G.S.) thanks CSIR for a research fellowship. The work is supported in part by grants from the Japan Society for the Promotion of Science (JSPS) and the Department of Science and Technology (DST), India.

${ }^{1}$ D. W. Oxtoby, Adv. Chem. Phys. 47, 487 (1981)

${ }^{2}$ D. W. Oxtoby, Annu. Rev. Phys. Chem. 32, 77 (1981).

${ }^{3}$ K. F. Herzfeld and T. A. Litovtiz, Absorption and Dispersion of Ultrasonic Waves (Academic, New York, 1959).

${ }^{4}$ J. L. Skinner, S. A. Egorov, and K. F. Everitt, in Ultrafast Infrared and Raman Spectroscopy, edited by M. Fayer (Marcel Dekker, New York, 2000), p. 675.

${ }^{5}$ B. J. Berne, M. E. Tuckerman, J. E. Straub, and A. L. R. Bug, J. Chem. Phys. 93, 5084 (1990).

${ }^{6}$ G. Goodyear, R. E. Larsen, and R. M. Stratt, Phys. Rev. Lett. 76, 243 (1996)

${ }^{7}$ M. E. Tuckerman and B. J. Berne, J. Chem. Phys. 98, 7301 (1993).

${ }^{8}$ M. Mishra and B. J. Berne, J. Chem. Phys. 103, 1160 (1995).

${ }^{9}$ K. Miyazaki, G. Srinivas, and B. Bagchi, J. Chem. Phys. 114, 6276 (2001).

${ }^{10}$ J. P. Boon and S. Yip, Molecular Hydrodynamics (Dover, New York, 1980).

${ }^{11}$ H. Mori, Prog. Theor. Phys. 33, 399 (1965), 33, 423 (1965), R. Zwanzig, in Lectures in Theoretical Physics, edited by W. E. Britten, B. W. Downs, and J. Downs (Springer-Verlag, New York, 1961), p. 135.

${ }^{12}$ L. Sjögren and A. Sjölander, J. Phys. C 12, 4369 (1979).

${ }^{13}$ G. F. Mazenko and S. Yip, in Modern Theoretical Chemistry, edited by B. J. Berne (Plenum, New York, 1977), Vol. 6, p. 181.

${ }^{14} \mathrm{~S}$. Chapman and T. Cowling, The Mathematical Theory of Non-Uniform Gases (Cambridge University Press, Cambridge, 1939).

${ }^{15}$ J. E. Straub, M. Borkovec, and B. J. Berne, J. Chem. Phys. 89, 4833 (1988). 